\title{
STRATEGIE INNOVATIVE PER IL CONTROLLO DI ZANZARE, VETTORI DI ORGANISMI PATOGENI
}

\author{
Nota del s.c. GIULIANO GASPERI (*)
}

(Adunanza del 29 giugno 2017)

SuNTO. - Le zanzare sono tra le specie di insetti più pericolose essendo vettori di agenti patogeni, responsabili delle malattie più diffuse, quali malaria, febbre dengue, chikungunya, febbre del Nilo occidentale, febbre gialla e Zika. Alcune zanzare dei generi Anopheles, Aedes e Culex sono responsabili di milioni di casi di infezioni umane, con centinaia di migliaia di morti all'anno. Ne derivano conseguenze drammatiche di carattere socio-economico e sanitario, specialmente in Paesi in via di sviluppo. Il controllo degli insetti nocivi è da tempo basato sull'uso prolungato degli insetticidi che inducono resistenza negli insetti e determinano inquinamento dell'ambiente. Nella seconda metà del XX secolo, la consapevolezza dell'importanza della salute umana e dell'ambiente ha stimolato lo sviluppo di nuovi approcci di controllo che hanno come target il comportamento riproduttivo. In particolare, la cosidetta Tecnica dell'Insetto Sterile (SIT), applicata con successo contro alcune specie di ditteri nocivi in diverse aree del mondo, ha rappresentato il punto di partenza per ricerche legate alla conoscenza dettagliata della biologia delle specie da combattere. Approcci di biologia molecolare hanno stimolato miglioramenti nell'applicazione di SIT mediante strategie di trasformazione genetica. Tecniche innovative quali RIDL, incompatibilità citoplasmatica (CI) e metodologie di gene drive rivestono un evidente interesse applicativo per il controllo/eradicazione delle zanzare.

$$
* * *
$$

ABSTRACT. - Mosquitoes are the most dangerous insect species being the vectors of the pathogens causing the most widespread diseases as Malaria, Dengue fever, Chikungunya, West Nile fever, Yellow fever, Zika. Several species of the Anopheles, Aedes and Culex are responsable for millions of human infections causing hundreds of thousands of deaths per year. This causes dramatic socio-economic and health conse-

(*) Dipartimento di Biologia e Biotecnologie "Lazzaro Spallanzani”, Università degli Studi di Pavia, Italy. E-mail: gasperi@unipv.it 
quences, especially in developing countries. Harmful insects are controlled using chemical insecticides which cause insecticide resistance and environmental pollution, due to long term use. In the second half of the $20^{\text {th }}$ century, new insect environmentally safe control stategies were been developed, based on the insect's reproductive behaviour. Notably, the Sterile Insect Technique (SIT) has been successfully applied against some dipteran pest species in several regions of the World. It drived several research efforts to increase our knowledge of the biology of the harmful species. SIT improvements have been also achieved with several molecular biology approaches and transgene technologies such as the innovative RIDL, cytoplasmic incompatibility (CI) and gene drive.

\section{INTRODUZIONE}

Gli insetti sono considerati gli animali di maggior successo evolutivo per la loro abbondanza e loro distribuzione in tutti gli ambienti, essendo in grado di adattarsi in modo efficace ad una grande varietà di habitat nei quali svolgono importanti funzioni ecologiche. Delle numerose specie di insetti descritte (1.5 milioni), molte sono vettori di agenti patogeni per animali e piante. Tra queste, le zanzare (famiglia Culicidae, Ordine Diptera), di cui sono state descritte circa 3.500 specie, rappresentano gli animali più pericolosi al mondo, essendo vettori di agenti patogeni (protozoi e virus). Essi sono tramessi dalle femmine infette all'ospite (uomo e/o vertebrato) mediante pasto di sangue, necessario per la maturazione delle uova. Tra le malattie più diffuse, trasmesse da alcune specie di zanzare dei generi Anopheles, Aedes e Culex, sono da menzionare malaria, febbre dengue, chikungunya, febbre del Nilo occidentale, febbre gialla e Zika. Anopheles, Aedes e Culex sono responsabili di milioni di infezioni umane, con centinaia di migliaia di morti all'anno $[1 ; 2]$. Queste patologie determinano drammatiche conseguenze di carattere socio-economico e sanitario, non solo in Paesi in via di sviluppo, ma anche in nuovi areali nel mondo, come conseguenza della globalizzazione dei traffici commerciali e dei cambiamenti climatici [3].

Tradizionalmente, le misure di controllo sono indirizzate a limitare l'esposizione dell'ospite alle zanzare mediante sostanze repellenti [4], insetticidi [5] o trattamenti medici [6]. Tuttavia, sebbene l'uso di insetticidi sia e sia stato per lungo tempo il più importante componente delle strategie di controllo delle popolazioni di zanzare, le malattie tramesse da zanzare stanno riacutizzandosi, generalmente a causa sia di resistenza agli insetticidi sviluppata nei vettori, che di resistenza dei patogeni. Numerosi studi hanno dimostrato che complessi meccanismi biochimici e genetici (in particolare, aumentata detossificazione metabolica degli 
insetticidi e diminuita affinità per proteine bersaglio) sono responsabili della resistenza agli insetticidi [7]. Sebbene gli insetticidi chimici siano estensivamente usati in tutti i paesi del mondo, un'aumentata consapevolezza sociale tende a sostituirli gradualmente con bioinsetticidi specifici, più sicuri per la salute umana. Si tratta di composti biologici efficaci, basati su tossine di origine microbica, con spiccata specificità per alcuni ordini di insetti [8]. Tuttavia, fenomeni di resistenza degli insetti anche ai bioinsetticidi sono stati recentemente riportati [9].

Il controllo degli insetti nocivi su base biologica rappresenta attualmente una delle maggiori sfide che la scienza e la società devono sostenere per far fronte a possibili emergenze, senza tuttavia disattendere il rispetto dell'ambiente. Nella seconda metà del XX secolo, sono state introdotte tecniche ecologicamente valide a seguito dell'intuizione della Tecnica dell'Insetto Sterile (SIT)[10] e dell'introduzione dei nemici naturali di specie nocive [11]. L'avvento poi delle metodologie del DNA ricombinante e dell'ingegneria genetica, accompagnate da maggiori conoscenze delle specie considerate, ha stimolato miglioramenti nell'applicazione di SIT.

Negli ultimi anni sono in atto ricerche innovative, mediante approcci di trasformazione genetica e di "gene editing", che sfruttano caratteristiche e fenomeni biologici, e tendono a ridurre drasticamente la potenzialità riproduttiva e la capacità vettoriale delle popolazioni $[12 ; 13 ; 14 ; 15 ; 16]$.

\section{LA TECNICA DELL'INSETTO STERILE (SIT)}

La Tecnica dell'Insetto Sterile è un metodo di controllo autocidico che sfrutta il comportamento riproduttivo degli insetti. Nella sua applicazione, SIT consiste nel rilascio sistematico di insetti sterilizzati, preferibilmente di sesso maschile, allevati in idonee biofabbriche. I maschi sterilizzati con mezzi fisici competono con quelli naturali per le femmine della popolazione bersaglio; di conseguenza, rilasciando continuamente maschi sterilizzati, il potenziale riproduttivo della popolazione bersaglio viene ridotto per la mancata produzione di progenie [17], teoricamente fino ad eradicazione della specie (Fig. 1).

L'efficienza di SIT dipende dalla possibilità di a) allevare in modo massivo la specie bersaglio; b) sterilizzare con radiazioni ionizzanti (raggi X, raggi g, neutroni) un notevole numero di insetti della 
specie bersaglio in un adatto stadio di sviluppo; c) rilasciare un notevole numero di insetti sterilizzati, ragionevolmente competitivi rispetto ai maschi selvatici; d) distribuire gli insetti sterilizzati con l'uso di sistemi economici nell'area da trattare; e) valutare accuratamente la dimensione delle popolazioni native prima e dopo il rilascio degli insetti sterilizzati; f) escludere l'eventuale immigrazione di femmine feconde nell'area trattata (che deve essere molto ampia e/o abbastanza isolata) [18].

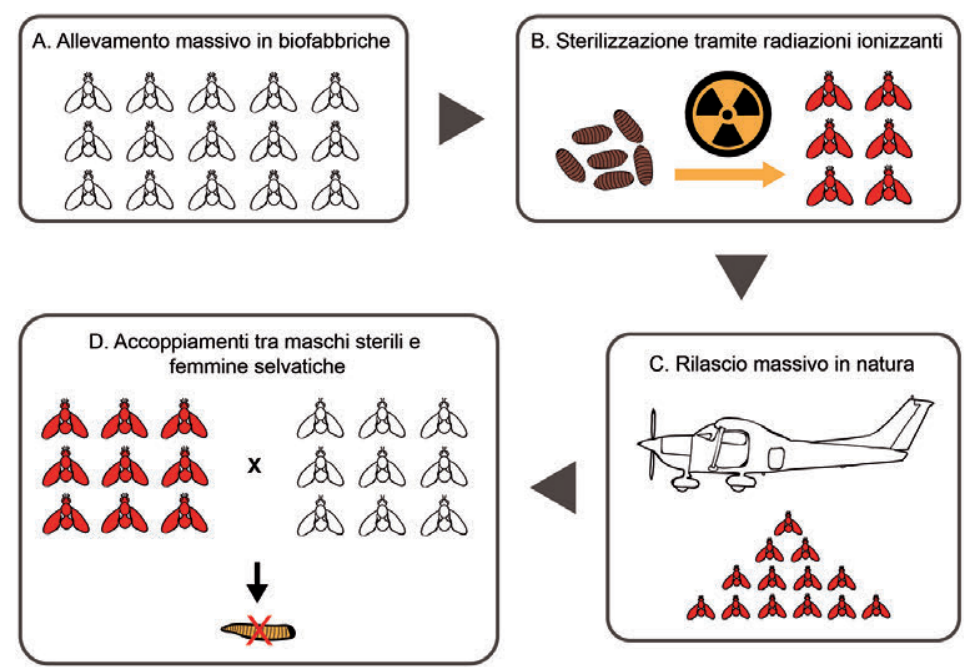

Fig. 1 - Applicazione della tecnica SIT (Tecnica dell'Insetto Sterile). A seguito di introduzione accidentale di una specie di insetto dannoso o di aumento della dimensione di una popolazione di una specie endemica, maschi sterilizzati della specie di interesse sono rilasciati in modo massivo nell' area bersaglio con opportuni mezzi di dispersione. I maschi sessualmente sterili, in numero superiore a quelli selvatici, si accoppiano con le femmine presenti nell'area, con il risultato che gli accoppiamenti non saranno fertili. Il risultato sarà un decremento della dimensione della popolazione che, se continuato nel tempo, porterà alla sua eradicazione.

Il concetto di SIT è stato sviluppato negli USA negli anni '30 del secolo scorso e la sua applicazione è stata realizzata con successo per la prima volta negli anni '50 contro Cochliomyia hominivorax (mosca assassina), dittero Calliphoridae, le cui larve si sviluppano come parassiti nei tessuti cutanei dei bovini, provocando pesanti miasi che causano perdite considerevoli all'allevamento di bestiame [10]. Il programma di eradicazione di questa specie mediante SIT è stato appli- 
cato per la prima volta con successo nel 1954 nell'isola di Curacao. Con questa tecnica $C$. hominivorax è stata eradicata dagli USA a partire dal 1982 e dall'America Centrale dal 2001. Una barriera permanente di mosche sterili viene tutt'ora mantenuta nel Darien gap di Panama allo scopo di prevenire reinfestazioni della specie dai paesi dell'America meridionale.

Lo sviluppo di questa tecnologia è stato in gran parte guidato da USDA (United States Department of Agriculture) e dalla divisione congiunta FAO/IAEA (Food and Agriculture Organization of the United Nations - International Atomic Energy Agency), enti coinvolti a livello sovranazionale nel contenimento e/o eradicazione di numerose specie di insetti dannosi. SIT è stato applicato con successo su popolazioni di ditteri tefritidi (Ceratitis capitata, Bactrocera dorsalis, Bactrocera cucurbitae, Bactrocera tryoni, Bactrocera oleae, Anastrepha suspensa, Anastrepha ludens), di ditteri calliforidi (Lucilia cuprina), di ditteri glossinidi (mosche tse-tse), di ditteri culicidi (Aedes aegypti) e di lepidotteri delle fam. Lymantriidae (Lymantrya dispar) e Gelechiidae (Pectinophora gossypiella, tacna del cotone) [19].

Dal momento che solo i maschi sterili contribuiscono alla soppressione della popolazione, un limite di questa tecnica è rappresentato dal concomitante rilascio di femmine sterilizzate: sebbene le uova deposte da queste femmine non siano in grado di svilupparsi in embrioni, poiché danneggiate a livello di DNA dalle radiazioni, le femmine mantengono il proprio abituale comportamento. Nel caso di insetti fitofagi, le femmine depongono le uova nell'ospite e, nel caso di femmine ematofaghe, queste continuano a nutrirsi di sangue agendo così da vettori di patogeni. La presenza di femmine nell'allevamento di massa, inoltre, raddoppia il costo dell'allevamento e, poiché talvolta può realizzarsi una sterilizzazione incompleta, sussiste il rischio di immettere nell'ambiente femmine fertili che potrebbero ridurre in modo significativo l'efficenza della tecnica SIT [20].

Sebbene la tecnica SIT sia stata spesso applicata utilizzando mosche sterilizzate di ambedue i sessi, attualmente si tende ad impiegare solamente ceppi di soli maschi ("Genetic Sexing Strains", GSSs) ottenuti mediante un approccio di genetica classica: questi ceppi presentano traslocazioni reciproche tra il cromosoma Y (tipico del sesso maschile) e un autosoma che porta un marcatore recessivo selezionabile. In questo modo i due sessi vengono discriminati in stadi precoci dello sviluppo con conseguente realizzazione di GSSs da immettere in 
natura [21]. E'noto che le radiazioni ionizzanti che portano a sterilizzazione tendono comunque ad abbassare la fitness dei maschi rilasciati; in base alla dimensione della popolazione da trattare con SIT, il rapporto dei maschi sterilizzati rispetto a quelli selvatici dovrebbe essere nel range di 1:2 - 1:15.

Le ricerche attualmente in atto sono intese a rendere la tecnica SIT più efficiente attraverso la generazione di ceppi di soli maschi mediante manipolazioni di genetica molecolare, e lo sfruttamento di particolari fenomeni biologici, anche quelli indotti negli insetti da microrganismi.

LA TECNICA RIDL

(RELEASE OF INSECTS CARRYING DOMINANT LETHALS)

Questa tecnica, descritta inizialmente in un lavoro condotto su Drosophila melanogaster, si basa sull'inserzione nel genoma dei maschi della specie di interesse di un gene letale dominante, mediante tecniche di biologia molecolare [22]. Nella formulazione originaria, il gene letale produce una proteina, chiamata tTAV (tetracycline transactivater variant), che si lega a un sito specifico nel genoma, chiamato TeTO (tetracycline operator), producendo una proteina tossica. L'accoppiamento della femmina con il maschio che porta il gene letale dominante (tTAV) determina così la morte della progenie nei primi stadi dello sviluppo. Se però gli insetti vengono allevati in presenza di tetraciclina (antidoto) assunta con il cibo, questo antibiotico si lega con la proteina tTAV inattivando il legame con TeTO, permettendo così la produzione di maschi e femmine vitali (condizione permissiva). Il gene letale dominante, e di conseguenza il sistema, è specifico per ciascuna specie di insetto di interesse [23]. Questa tecnica è stata sviluppata e brevettata da una ditta privata (Oxitec Ltd, Oxford Insect Technologies).

La condizione permissiva è assente in natura, e pertanto gli insetti omozigoti per il gene dominante letale, accoppiandosi con gli insetti selvatici, generano una progenie eterozigote che muore [24]. Il rilascio periodico di maschi con gene letale rappresenta un sistema efficiente di soppressione delle popolazioni di insetti basato su SIT. Rispetto alla tecnica classica SIT, RIDL ha il vantaggio che la sterilizzazione non richiede radiazioni ionizzanti e pertanto la competitività dei maschi è 
mantenuta [25]. Tuttavia, il controllo in campo con il sistema RIDL contro Aedes aegypti, condotto con successo in diverse aree dove Dengue è endemica (Isole Cayman, Malesia e Brasile), ha richiesto per sicurezza la sterilizzazione dei maschi prima del rilascio in modo tale che il gene modificato non potesse diffondersi fra le generazioni successive $[15 ; 26]$.

\section{CONTROLLO BIOLOGICO MEDIANTE INCOMPATIBILITÀ CITOPLASMATICA (CI)}

Si tratta di un fenomeno indotto da un gruppo di batteri gramnegativi del genere Wolbachia, trovati come endosimbionti nei tessuti riproduttivi degli Artropodi e di altri organismi [27]. Questi batteri sono in grado di manipolare, con vari meccanismi, la riproduzione dei loro ospiti, inducendo fenomeni quali incompatibilità riproduttiva (CI), partenogenesi e femminilizzazione dei maschi. L'incompatibilità citoplasmatica (CI) rappresenta il fenomeno più interessante per strategie di controllo dal momento che questa caratteristica viene ereditata per via materna con modalità uni- o bi-direzionale (Fig. 2). Nella incompatibilità uni-direzionale, l'incrocio tra un maschio infetto con Wolbachia e una femmina non-infetta dà origine ad uno zigote nonvitale. L'incrocio reciproco è invece fertile. Wolbachia induce incompatibilità nei maschi, mentre nelle femmine ristabilisce la compatibilità. Nella incompatibilità bi-direzionale, dove maschi e femmine portano differenti ceppi di Wolbachia, tutti gli incroci eterozigoti sono incompatibili. Essendo stato dimostrato che Wolbachia è in grado di diffondersi con estrema rapidità in popolazioni non-infette [28], questa caratteristica aggiunge un'ulteriore vantaggio nei programmi di controllo mediato da questo simbionte.

Oltre ad agire come un vero manipolatore della riproduzione dell'ospite, Wolbachia presenta alcuni ceppi in grado anche di inibire la replicazione di virus e protozoi nelle zanzare [29; 30;31]. Queste condizioni rappresentano un potente mezzo per il controllo di popolazioni infette di specie di Aedes [32] e di Anopheles [33].

Con particolari tecniche biotecnologiche è possibile trasferire ceppi di Wolbachia da una specie all'altra, generando nuovi pattern di CI ed estendendo l'elenco di specie da controllare con strategie basate su Wolbachia. La tecnologia usata allo scopo si basa su microiniezione 
di ooplasma infetto (transinfezione) contente l'appropriato ceppo di Wolbachia in embrioni recipienti [34]. Questa strategia di controllo è stata recentemente usata con successo contro popolazioni di Aedes aegypti in Australia e Vietnam [15], e prove di campo sono state condotte in Italia contro Aedes albopictus [35].

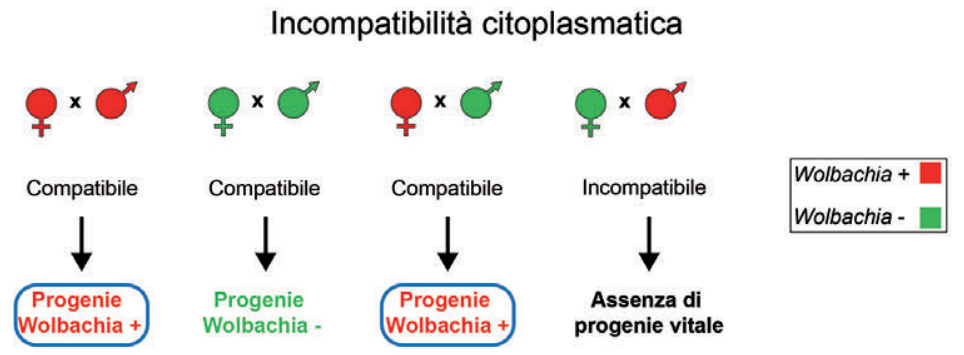

Incompatibilità citoplasmatica bidirezionale

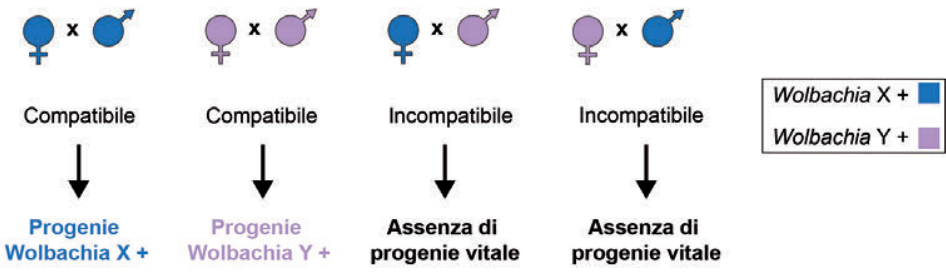

Fig. 2 - L'incompatibilità citoplasmatica (CI) è un fenomeno che impedisce agli spermatozoi e alle nova di formare una prole vitale. L'effetto deriva dai cambiamenti nelle cellule del gamete causati da Wolbachia. a) CI uni-direzionale si realizza con femmine in grado di accoppiarsi con successo sia con maschi non-infetti che con maschi infetti con lo stesso ceppo di Wolbachia; b) CI bi-direzionale può realizzarsi in maschi e femmine infetti con due differenti ceppi di Wolbachia, incapaci di generare progenie fertile.

\section{TECNOLOGIE DI INGEGNERIA GENETICA: GENE DRIVE}

A differenza delle strategie soprariportate che necessitano di introduzioni continue di maschi sterili nelle popolazioni bersaglio, un promettente approccio di manipolazione genetica, il cosidetto "genedrive”, ha recentemente dimostrato la possibilità di selezionare alcune 
caratteristiche ereditarie, nella fattispecie quelle che rendono sterili le femmine di zanzare o che ne riducono le possibilità di accoppiamento, o ancora che le rendono immuni al patogeno, e far sì che queste caratteristiche si diffondano rapidamente alle generazioni successive [36; 37]. In realtà "gene drive" è un fenomeno naturale, dovuto ad elementi genetici in grado di manipolare la gametogenesi e la riproduzione, e in modo tale da aumentare la propria trasmissione alle generazioni successive, fino a quando questi particolari elementi genetici non siano presenti in ogni discendente [38]. Questo fenomeno deriva da una varietà di meccanismi utilizzati nelle manipolazioni genetiche della linea germinale per far propagare una serie di geni in una popolazione, alterando la probabilità che un allele specifico venga trasmesso alla progenie dalla naturale probabilità del 50\% (distorsione del sesso). Sistemi di gene drive altamente invasivi possono determinare una deviazione ereditaria vicino al 100\%. Infatti, assumendo una popolazione con accoppiamenti random e senza costo di fitness, un elemento gene drive con $100 \%$ di trasmissione alla progenie aumenterebbe la sua frequenza fino a 0.99 dopo soltanto poche generazioni [39; 40].

Elementi di gene drive in natura sono rappresentati da elementi trasponibili (TEs), da microrganismi ereditabili e da endonucleasi di riferimento (homing endonucleases, HEGs) che tendono a diffondersi in una popolazione bersaglio per più generazioni, anche quando sono rilasciati a basse frequenze [14]. In particolare, HEGs si sono rivelate uno strumento ideale per distruggere geni essenziali per la fertilità della femmina, per la determinazione del sesso, per il riconoscimento dell'ospite o per la trasmissione del patogeno (knock-out scheme) e per diffondere modificazioni deleterie che innescano la soppressione della popolazione (knock-in scheme). HEGs sono localizzate entro la sequenza di riconoscimento del DNA, che lo rende resistente al taglio. Quando HEG viene in contatto con il suo cromosoma omologo intatto in cellule eterozigoti, esso taglia la sequenza bersaglio. Come risultato del processo di riparo del cromosoma (homology direct repair mechanism, HDR), indotto dalla rottura del doppio filamento, HEG può essere copiato convertendo le cellule eterozigoti in omozigoti. Questo meccanismo, noto come "homing", promuove un rapido aumento di frequenza di HEG in una popolazione [14].

Nell'ultimo decennio sono state proposti diversi sistemi artificiali di homing/gene drive basati su nucleasi modulari, di cui uno dei più promettenti è stato realizzato mediante editing genico CRISPR-Cas9. 
Questo sistema di gene drive sintetico è stato designato in modo tale da essere attivo nella linea germinale di Anopheles gambiae, e in modo da essere capace di distruggere geni essenziali per la fertilità della femmina, come il gene di fertilità AGAP007280 [41]. In gene drive mediato da CRISPR, il costrutto comprende una singola nucleasi Cas9 e una guida di RNA che determina la specificità del taglio del sito bersaglio. Quando inserito nel genoma ed espresso nella linea germinale, la proteina Cas9 distrugge il gene AGAP007280. In risposta alla rottura del doppio filamento, la cellula impiega il sistema HDR (homology direct repair mechanism) per copiare il costrutto che include qualunque carico genetico nel cromosoma omologo intatto. Sebbene singole femmine di zanzara con una sola copia del gene difettivo sono ancora fertili, il sistema gene drive assicura l'ereditarietà della mutazione nel 99-95\% della progenie ed un rapido aumento di frequenza di AGAP007280 nella popolazione. Come risultato, la progenie ha un'alta probabilità di ricevere una copia del costrutto gene drive da ambedue i genitori: tutte le femmine omozigoti per la mutazione sono sterili, mentre i maschi continuano a trasmettere il gene drive. In tal modo, sono richieste poche generazioni per diffondere il tansgene/mutazione nelle popolazioni selvatiche a partire da bassa frequenza fino a raggiungere quasi la fissazione (Fig. 3).

Questa tecnologia ha le potenzialità per indurre un sostenibile decremento di densità di zanzare imponendo un carico riproduttivo sulla popolazione, anche se è stato osservata forte pressione selettiva per alleli resistenti come variazioni di sequenza al sito bersaglio di endonucleasi Cas9. Tuttavia, costrutti sintetici gene drive indirizzati a sequenze altamente conservate nel genoma decrescono la probabilità di resistenza [42].

L'eliminazione del gene doublesex (Agdsx) mediante il sistema CRISPR-Cas9 ha portato per la prima volta alla soppressione completa si popolazioni di Anopheles gambiae tenute in piccole gabbie dopo 7-11 generazioni (Andrea Crisanti, comunicazione personale). Il gene $A g d x s$ è altamente conservato e codifica due trascritti alternativamente espressi, $A g d s x \mathrm{~F}$ (dsx-femminile) e $A g d s x \mathrm{M}$ (dsx-maschile). Questo gene determina il dimorfismo sessuale nelle zanzare. L'eliminazione mediata da CRISPR-Cas9 della sequenza tra l'introne 4 e l'esone 5 in Agdsx inibisce la formazione del trascritto funzionale $A g d s \times \mathrm{F}$ e induce sterilità nelle femmine, ma non influenza la fitness dei maschi. Femmine omozigoti nulle $\left(\mathrm{dsxF}^{-/}\right)$mostrano intersessi con caratteristiche maschio- 
specifiche, quali antenne piumose, proboscide anormale e pterigopodi non ruotati. Queste femmine sono incapaci di pungere, di nutrirsi su sangue e di deporre uova. Dati i limiti funzionali del $A g d x s$, dopo 14 generazioni di zanzare tenute in piccole gabbie non è stata osservata selezione di alleli resistenti. Esperimenti in grandi gabbie che riproducano condizioni di ambienti tropicali sono in atto presso l'Imperial College London e il Polo d'Innovazione di Genomica Genetica e Biologia (sede di Terni) con lo scopo di valutare la dinamica di popolazione e il potenziale di soppressione di questo costrutto di gene drive sviluppato in zanzare del genere Anopheles.
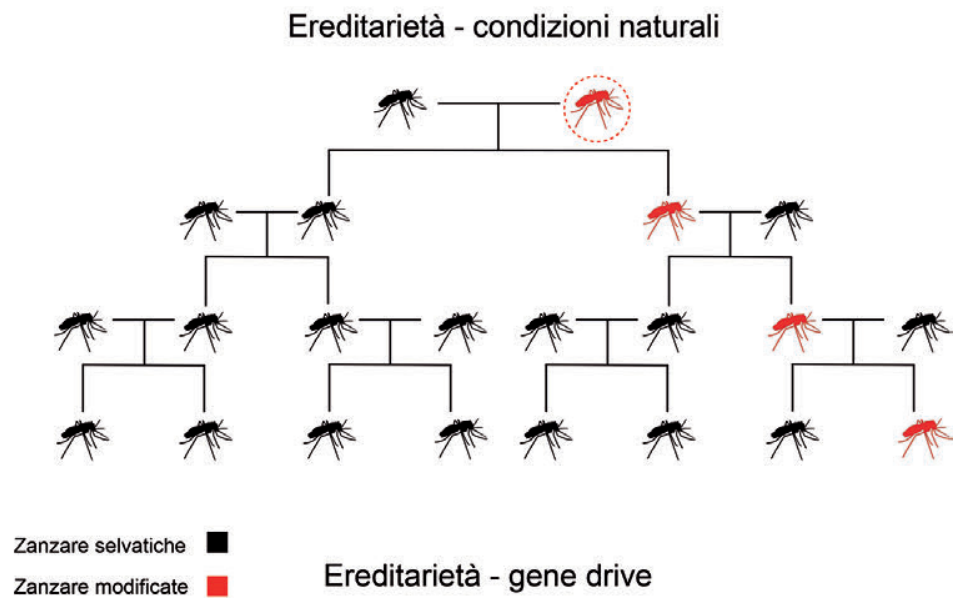

Ereditarietà - gene drive

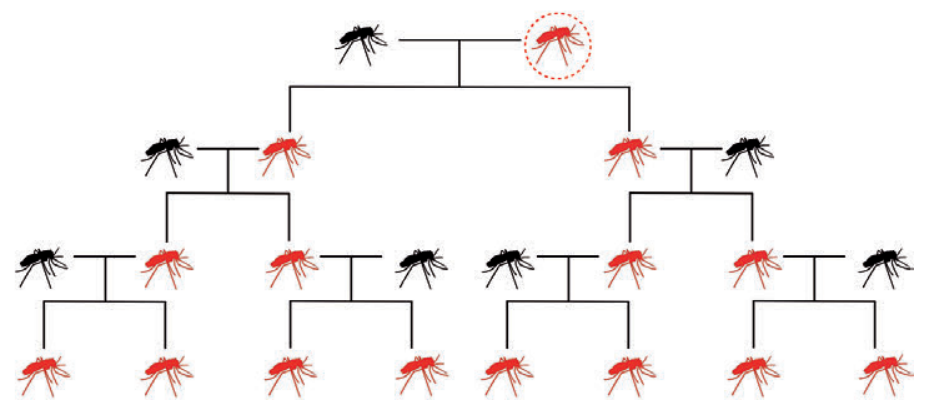

Fig. 3 - Le zanzare e altri organismi trasmettono un dato carattere alle generazioni successive con probabilità del 50\% (eredità mendeliana), mentre un sistema gene drive copia e inserisce se stesso nei cromosomi di ambedue i genitori, assicurando una probabilità della sua trasmissione fino al $100 \%$. 


\section{CONCLUSIONI}

Le strategie di controllo genetico sfruttano il comportamento riproduttivo degli insetti, attraverso l'accoppiamento, con l'uso di ceppi di soli maschi capaci di disperdersi attivamente nell'ambiente alla ricerca delle femmine selvatiche. Le strategie genetiche sono tipicamente specie-specifiche. Il controllo genetico non deve tuttavia essere visto come la panacea capace di eliminare da solo popolazioni di insetti nocivi ma, opportunamente integrato con tutti i metodi convenzionali, potrebbe portare a risultati efficienti.

Mentre alcune delle strategie, quali il classico SIT, sono applicate in campo in modo regolare a seguito di approvazione delle normative regolatorie vigenti [43], tutte quelle che fanno uso di insetti geneticamente modificati rispettano al momento il principio di precauzione. Alcuni Paesi (Isole Cayman, Malesia e Brasile, Australia e Vietnam) hanno dato autorizzazioni per usare la tecnica RIDL modificata o SIT integrata con Wolbachia [13; 24], mentre i ceppi di soli maschi generati mediante le promettenti manipolazioni di gene editing sono al momento trattati in ambienti confinati (Andrea Crisanti, comunicazione personale).

L'uso di insetti geneticamente manipolati da immettere in natura per il controllo/eradicazione di specie nocive, è argomento di opportune normative $[44 ; 45 ; 46]$ a fronte di un forte dibattito a livello nazionale e internazionale relativamente alle tematiche della protezione dell'ambiente e della salute, nonostante ci sia ampio consenso in ambito scientifico nel ritenere che gli insetti geneticamente modificati non presentino rischi all'ambiente [47].

\section{RINGRAZIAMENTI}

Si ringrazia la Dr Francesca Scolari per la realizzazione delle figure incluse in questo scritto. 


\section{BIBLIOGRAFIA}

1. Hotez P.J., Fenwick A., Savioli L., Molyneux D.H. Rescuing the bottom billion through control of neglected tropical diseases. Lancet (2009) 373: 1570-1575.

2. Mayer S.V., Tesh R.B., Vasilakis N. The emergence of arthropod-borne viral diseases: a global prospective on dengue, chikungunya and zika fevers. Acta Trop. (2017) 166: 155-163.

3. Reiter P. Climate change and mosquito-borne disease. Environ. Health Persp. (2001) 109(suppl 1): 141-161. doi:10.1289/ehp.01109s1141

4. Paluch G., Bartholomay L., Coats J. Mosquito repellents: a review of chemical structure diversity and olfaction. Pest Manag. Sci. (2010) 66: 925-935.

5. Rivero A., Vézilier J., Weill M., Read A.F., Gandon S. Insecticide Control of Vector-Borne Diseases: When Is Insecticide Resistance a Problem? PLoS Path (2010) 6: e1001000. doi:10.1371/journal.ppat.1001000

6. Utzinger J., Becker S., Knopp S., Blum J. Neumayr A., Keiser J., Hatz C. Neglected tropical diseases: diagnosis, clinical management, treatment and control. Swiss Medical Weekly (2012) doi:10.4414/smw.2012.13727

7. Liu N. Insecticide Resistance in Mosquitoes: Impact, Mechanisms, and Research Directions. Annu. Rev. Entomol (2015) 60:537-559. doi:10.1146/annurev-ento010814-020828

8. Mazid S., Kalita J.C., Rajkhowa R.C. A review on the use of biopesticides in insect pest management. Int. J. Sci. Adv. Technol. (2011) 1: 169-178.

9. Tabashnik B.E., Brévault T., Carrière Y. Insect resistance to Bt crops: lessons from the first billion acres. Nature Biotech. (2013): 31: 510-521. doi:10.1038/nbt.2597

10. Knipling E. F. Possibilities of insect control or eradication through the use of sexually sterile males. J. Econ. Entomol. (1955) 48: 459-462.doi:10.1093/jee/ 48.4.459

11. Barratt B.I.P., Moran V.C., Bigler F., van Lenteren J.C. The status of biological control and recommendations for improving uptake for the future. BioControl (2017) 63: 155-167. doi:10.1007/s10526-017-9831-y

12. Scolari F., Siciliano P., Gabrieli P., Gomulski L. M., Bonomi A., Gasperi G., Malacrida A.R. Safe and fit genetically modified insects for pest control: from lab to field applications. Genetica (2011) 139: 41-52. doi: 10.1007/s10709-0109483-7

13. Scolari F., Gomulski L.M., Gabrieli P., Manni M., Savini G., Gasperi G., Malacrida A.R.. How the 'omics' era will impact fruit fly pest control: the example of the Mediterranean fruit fly, Ceratitis capitata. BMC Genetics (2014) 15 (Suppl 2): S11.

14. Burt A. Site-specific selfish genes as tools for the control and genetic engineering of natural populations. Proc. Royal Soc. B: Biol. Sci. (2003) 270: 921-928. doi:10.1098/rspb.2002.2319

15. Alphey L. Genetic Control of Mosquitoes. Annu. Rev. Entomol. (2014) 59: 205 224. doi:10.1146/annurev-ento-011613-162002 
16. Galizi R., Hammond A., Kyrou K., Taxiarchi C., Bernardini F., O'Loughlin S.M., Papathanos P.A., Nolan T., Windbichler N., Crisanti A. A CRISPR-Cas9 sex-ratio distortion system for genetic control. Sci. Rep. (2016) 6: 31139. doi:10.1038/srep31139

17. Hendrichs J., Robinson A. Sterile Insect Technique. pp. 953-957. In Encyclopedia of Insects (Resh V., Cardé R. Eds.) pp. 1168. Academic Press, Elsevier Publ. (2009). doi:10.1016/b978-0-12-374144-8.00252-6

18. Vreysen M.J.B., Robinson A.S., Hendrichs J. (Eds.) Area-Wide Control of Insect Pests: From Research to Field Implementation. pp 789. Springer Publ., 3300 AA Dordrecht, The Netherlands (2007).

19. Pereira R., Yuval B., Liedo P. Teal P.E.A., Shelly T.E., McInnis D.O., Hendrichs $\mathrm{J}$. Improving sterile male performance in support of programmes integrating the sterile insect technique against fruit flies. J. Appl. Entomol (2011) 137: 178-190. doi:10.1111/j.14390418.2011.01664.x

20. McInnis D.O., Tam S., Grace C., Miyashita D. Population suppression and sterility rates induced by variable sex ratio, sterile insect releases of Ceratitis capitata (Diptera: Tephritidae) in Hawaii. Ann. Entomol. Soc. Am. (1994) 87: 231-240. doi:10.1093/aesa/87.2.231

21. Franz G. Genetic sexing strains in Mediterranean fruit fly, an example for other species amenable to large-scale rearing for the sterile insect technique. pp. 427 451. In: Sterile Insect Technique. Principles and Practice in Area-Wide Integrated Pest Management (Dyck V.A., Hendrichs J., Robinson A.S. Eds.) pp. 787. Springer Publ., The Netherlands (2005).

22. Thomas D.D., Donnelly C.A., Wood R.J., Alphey L.S. Insect Population Control Using a Dominant, Repressible, Lethal Genetic System. Science (2000) 287: 2474-2476. doi:10.1126/science.287.5462.2474

23. Harvey-Samuel T., Ant T., Alphey L. Towards the genetic control of invasive species. Biol. Invasions (2017) 19: 1683-1703. doi:10.1007/s10530-017-1384-6

24. Gong P., Epton M.J., Fu G., Scaife S., Hiscox A., Condon K. C., Condon G.C., Morrison N.I., Kelly D.V., Dafa'alla T., Coleman P.G., Alphey L. A dominant lethal genetic system for autocidal control of the Mediterranean fruitfly. Nature Biotech. (2005) 23: 453-456.

25. Black W.C., Alphey L., James A.A. Why RIDL is not SIT. Trends Parasitol. (2011) 27: 362-370. doi:10.1016/j.pt.2011.04.004

26. Carvalho D.O., McKemey A.R., Garziera L., Lacroix R., Donnelly C.A., Alphey L., Malavasi A., Capurro M.L. Suppression of a field population of Aedes aegypti in Brazil by sustained release of transgenic male mosquitoes. PLoS Negl. Trop. Dis. (2015) 9: e0003864. doi:10.1371/journal.pntd.0003864

27. Werren J.H., Baldo L., Clark M.E. Wolbachia: master manipulators of invertebrate biology. Nat. Rev Microbiol. (2008) 6: 741-751.

28. Turelli M. Evolution of incompatibility inducing microbes and their hosts. Evolution (1994) 48: 1500-1513.

29. Moreira L.A., Iturbe-Ormaetxe I., Jeffery J.A., Lu G., Pyke A.T., Hedges L.M., Rocha B.C., Hall-Mendelin S., Day A., Riegler M., Hugo L.E., Johnson K.N., 
Kay B.H., McGraw E.A., van den Hurk A.F., Ryan P.A., O’Neill S.L. A Wolbachia symbiont in Aedes aegypti limits infection with Dengue, Chikungunya, and Plasmodium. Cell (2009) 139: 1268-1278. doi:10.1016/ j.cell.2009.11.042

30. Walker T., Johnson P.H., Moreira L.A., Iturbe-Ormaetxe I., Frentiu F.D., McMeniman C.J., Leong Y.S., Dong Y., Axford J., Kriesner P., Lloyd A.L., Richie S.A., O’Neill S.L., Hoffmann A.A. The wMel Wolbachia strain blocks dengue and invades caged Aedes aegypti populations. Nature (2011) 476: 450453. doi:10.1038/nature10355

31. Sinkins S.P. Wolbachia and arbovirus inhibition in mosquitoes. Future Microbiol. (2013) 8: 1249-1256.

32. Iturbe-Ormaetxe I., Walker T., O' Neill S.L. Wolbachia and the biological control of mosquito-borne disease. EMBO rep (2011) 12: 508-518. doi:10.1038/embor. 2011.84 .

33. Bian G., Joshi D., Dong Y., Lu P., Zhou G., Pan X., Xu Y., Dimopoulos G., Xi Z Wolbachia invades Anopheles stephensi populations and induces refractoriness to Plasmodium infection. Science (2013) 340:748-751. doi: 10.1126/science. 1236192

34. Hughes G.L., Rasgon J.L. Transinfection: a method to investigateWolbachiahost interactions and control arthropod-borne disease. Insect Mol. Biol. (2013) 23: 141-151. doi:10.1111/imb.12066

35. Puggioli A., Calvitti M., Moretti R., Bellini R. wPip Wolbachia contribution to Aedes albopictus SIT performance: advantages under intensive rearing. Acta Trop. (2016) 164: 473-481. doi.org/10.1016/j.actatropica.2016.10.014

36. Windbichler N., Papathanos P.A., Crisanti A. Targeting the X chromosome during spermatogenesis induces $\mathrm{Y}$ chromosome transmission ratio distortion and early dominant embryo lethality in Anopheles gambiae. PLoS Genet. (2008) 4: e1000291. doi:10.1371/journal.pgen.1000291

37. Champer J., Buchman A., Akbari O.S. Cheating evolution: engineering gene drives to manipulate the fate of wild populations. Nature Rev. Genet. (2016) 17: 146-159. doi:10.1038/nrg.2015.34

38. Sandler L., Novitski E. Meiotic Drive as an Evolutionary Force. Am. Nat. (1957) 91: 105-110. doi:10.1086/281969.

39. Curtis C.F. Possible use of translocations to fix desirable genes in insect pest populations. Nature (1968) 218: 368-369.

40. Akbari O.S., Bellen H.J., Bier E., Bullock S.L., Burt A., Church G.M., Cook K.R., Duchek P., Edwards O.R., Esvelt K.M., Gantz V.M., Golic K.G., Gratz S.J., Harrison M.M., Hayes K.R., James A.A., Kaufman T.C., Knoblich J., Malik H.S., Matthews K.A., O’Connor-Giles K.M., Parks A.L., Perrimon N., Port F., Russell S., Ueda R., Wildonger J. Safeguarding gene drive experiments in the laboratory. Science (2015) 349: 927-929. doi:10.1126/science.aac7932

41. Hammond A., Galizi R., Kyrou K., Simoni A., Siniscalchi C., Katsanos D., Gribble M., Baker D., Marois E., Russell S., Burt A., Windbichler N., Crisanti A., Nolan T.A. CRISPR-Cas9 gene drive system targeting female reproduction 
in the malaria mosquito vector Anopheles gambiae. Nature Biotech. (2016) 34: 78-83. doi: $10.1038 /$ nbt.3439

42. Hammond A.M., Kyrou K., Bruttini M., North A., Galizi R., Karlsson X. Kranjc N., Carpi F.M., D'Aurizio R., Crisanti A., Nolan T. The creation and selection of mutations resistant to a gene drive over multiple generations in the malaria mosquito. PLoS Genet. (2017) 13: e1007039. doi: 10.1371/journal.pgen. 1007039.

43. IAEA (International Atomic Energy Agency) Sterile insect technique. https://www.iaea.org/topics/sterile-insect-technique, Vienna, Austria

44. Marshall J.M. The Cartagena Protocol and genetically modified mosquitoes. Nature Biotech. (2010) 28: 896-89.

45. Reeves R.G., Denton J.A., Santucci F., Bryk J., Reed F.A. Scientific standards and the regulation of genetically modified Iinsects. PLoS Negl Trop Dis (2012) 6: e1502. doi:10.1371/journal.pntd.0001502

46. WHO-TDR, FNIH. Guidance framework for testing of genetically modified mosquitoes. pp. 132. World Health Organization, Geneva (2014).

47. Reis-Castro L. Genetically modified insects as a public health tool: discussing the different bio-objectification within genetic strategies. Croatian Med. J. (2012) 53: 635-638. doi:10.3325/cmj.2012.53.635 